\title{
Avaliação de um protocolo clínico por enfermeiros no tratamento da sepse
}

\section{Evaluation of a clinical protocol by nurses in sepse treatment}

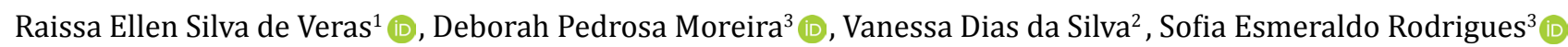 \\ 1. Discente do curso de Enfermagem pelo Centro Universitário Christus (UNICHRISTUS), Fortaleza, CE, Brasil. 2. Docente do Curso de Centro Universitário \\ Christus (UNICHRISTUS), Fortaleza, CE, Brasil. 3. Enfermeira do Hospital São Carlos, Fortaleza, CE, Brasil.
}

\section{Resumo}

Objetivo: avaliar o uso de um protocolo clínico por enfermeiros no tratamento da sepse em um hospital particular. Metodologia: estudo do tipo descritivo com abordagem qualitativa realizado em um hospital de nível terciário com enfermeiros da emergência e unidades de terapia intensiva totalizando 14 participantes, no período de outubro e novembro de 2018. Utilizou-se para a coleta de dados um gravador e um formulário com perguntas semiestruturadas, avaliadas por meio da análise de conteúdo. Resultados: foram elaboradas quatro categorias intituladas: capacitação dos profissionais para manejo do protocolo; conhecimento acerca do protocolo sepse; desafios do enfermeiro no uso do protocolo; experiências exitosas: desfecho do paciente pós-protocolo. Conclusão: por meio deste estudo, foi possível evidenciar a atuação do enfermeiro diante de pacientes com quadro séptico. Nessa assistência, incidem ainda os desafios que eles enfrentam para que o processo tenha início e fim, e não seja interrompido por qualquer eventualidade, como demora dos serviços acionados. Reforça que o protocolo não é apenas mais um documento da assistência, e sim uma ferramenta importante para prestar o melhor cuidado de enfermagem, que causa impacto em custos hospitalares e melhoria da imagem do hospital no mercado.

Palavras-chave: Protocolos Clínicos. Sepse. Enfermagem.

\begin{abstract}
Objective: To evaluate the use of a clinical protocol by nurses in the treatment of sepsis in a private hospital. Methodology: a descriptive study with a qualitative approach performed in a tertiary-level hospital with emergency nurses and intensive care units totaling 14 participants, in the period of October and November, 2018. A recorder and form with semi structured questions, analyzed through content analysis. Results: it was structured in four categories entitled "Professional qualification for protocol management"; knowledge about sepsis protocol; challenges of the nurse in the use of protocol; successful experiences: post-protocol patient outcome. Conclusion: through this study it was possible to evidence the nurse's performance in patients with septic syndrome. This assistance also addresses the challenges they face in order for the process to begin and end, and not be interrupted by any eventuality, as a delay in the services involved. It reinforces that the protocol is not only a document of care, but an important tool to provide the best nursing care, which causes impact on hospital costs and improvement of the hospital's image in the market.
\end{abstract}

Key words: Clinical Protocols. Sepsis. Nursing.

\section{INTRODUÇÃO}

Segundo o Instituto Latino Americano de Sepse (ILAS), a sepse consiste em uma infecção suspeita ou confirmada associada à disfunção orgânica ameaçadora à vida em decorrência da presença de resposta desregulada à infecção. As nomenclaturas de Síndrome de Reposta Inflamatória Sistêmica (SIRS), infecção sem disfunção, sepse e choque séptico indicam o desencadeamento da infecção notificada, essas delimitações auxiliam na deteç̧ão precoce dos casos que, associado à instituição do adequado tratamento, implica a regressão do quadro infeccioso ${ }^{1}$.

A sepse é uma condição de saúde muito comum em unidades de cuidados intensivos, com alta prevalência nos países desenvolvidos, e no Brasil, está entre as taxas mais altas. Nos Estados Unidos da América (EUA), por exemplo, cerca de 3\% dos pacientes internados têm sepse e parte deles são tratados em Unidades de Terapia Intensiva (UTI). Das admissões realizadas nessas unidades nos EUA, a causa por sepse gira em torno de $10 \%$, entre outras. Embora sua prevalência seja um grande desafio para a saúde na perspectiva mundial, pesquisas apontam que a mortalidade por esse agravo vem reduzindo desde o final da década de 70 nos EUA e na Europa. Porém, não há relatos favoráveis ao Brasil com relação à redução da mortalidade por sepse ${ }^{2-3}$.

Um estudo retrospectivo realizado por Taniguchi et al $(2017)^{3}$ investigou a epidemiologia das mortes por sepse no Brasil, utilizando dados secundários do Sistema Brasileiro de Informação sobre Mortalidade (SIM), entre os anos de 2002 a 2010. Os achados revelam que o número de mortes por sepse aumentou durante o período analisado, de 69,5 óbitos por 100 000 para 97,8 óbitos por 100000 habitantes.

Estima-se que, no Brasil, existam aproximadamente 600 mil 
novos casos de sepse a cada ano, que geram um grande impacto nos indicadores de morbimortalidade, pois as consequências da sepse são responsáveis por cerca de 250 mil mortes/ano, tornando-se um verdadeiro desafio para a saúde da população brasileira ${ }^{4}$.

Além de levar ao óbito, a sepse pode causar sequelas cognitivas significantes naqueles que sobrevivem ao quadro, comprometendo sobremaneira a qualidade de vida. Segundo uma pesquisa realizada nos EUA, há outra consequência da sepse que seria o aumento nos custos hospitalares, sendo esses maiores em lactentes, nos não sobreviventes, nos pacientes de UTI, nos pacientes cirúrgicos e naqueles com falência de mais de um órgão ${ }^{5-6}$.

Com isso, a implementação de um protocolo clínico voltado para o manejo da sepse é de suma importância, pois conduzem às ações a fim de se obter um alto nível de eficiência na assistência, gerando impacto na sobrevivência dos pacientes acometidos com a doença, assim como também, na redução do tempo de internação hospitalar e nas taxas de morbidade e mortalidade, já que este documento pode ser acionado para pacientes que apenas apresentem suspeita de sepse ${ }^{7}$.

O processo de construção de um protocolo assistencial deve respeitar os princípios legais e éticos da profissão e ser baseado em evidências. Ele possui vantagens como: redução da variedade do cuidado, segurança para usuários e profissionais de saúde, empodera para a tomada de decisão, subsídio para a elaboração de indicadores de processo e de resultado, comunicação entre a equipe multiprofissional, coordenação do cuidado ${ }^{8}$.

A atuação da enfermagem é imprescindível no gerenciamento do protocolo clínico de sepse, pois é a equipe que está mais próxima do paciente durante todo o cuidado, desde sua admissão à unidade hospitalar até a alta, e pode detectar precocemente seus sinais e sintomas. A equipe deve ser devidamente treinada para o reconhecimento suspeito ou confirmado de sepse, o enfermeiro deve conduzir a avaliação de forma sistematizada, utilizando-se da ferramenta processo de enfermagem, em que a primeira etapa consiste na coleta de dados, em que a anamnese e o exame físico são fundamentais para o diagnóstico precoce de sepse e direcionam de forma objetiva o cuidado de enfermagem ${ }^{9-10}$.

É relevante a adoção do protocolo clínico de sepse pela instituição, pois há um empoderamento da equipe de enfermagem no seu manejo, visto que o enfermeiro, ao perceber sinais e sintomas que sugerem quadro de sepse no paciente, pode acioná-lo imediatamente. Há também um melhor direcionamento do cuidado por todos os envolvidos no processo, pois, devido ao protocolo, o atendimento é realizado em tempo hábil, os exames e administração dos medicamentos acontecem também de forma mais rápida ${ }^{11}$.

Diante do exposto, este artigo objetiva avaliar o uso de um protocolo clínico por enfermeiros no tratamento da sepse em um hospital particular.

\section{MÉTOdOS}

Trata-se de um estudo do tipo descritivo com abordagem qualitativa, realizado em um hospital da rede particular de nível terciário, referência em transplante hepático, que é um dos mais renomados na cidade de Fortaleza- $C E$, no período de Outubro e Novembro de 2018.

Para a determinação dos participantes, utilizou-se o critério de saturação dos dados, ou seja, quando os discursos se tornarem repetitivos. Nos setores de emergência e UTI atuam 22 enfermeiros, dos quais 14 resultaram na amostragem. A escolha dos setores supracitados se deu por conta de que a sepse ocorre mais em UTI, conforme estudos, e na emergência, devido a representar o setor de maior movimentação de usuários por ser porta de entrada do hospital; com isso, os profissionais tendem a se deparar com mais frequência com quadros de sepse.

Foram incluídos os enfermeiros que atuam nas unidades de terapia intensiva e na emergência; os que fazem uso do protocolo clínico de sepse; e os que possuem experiência acima de seis meses de uso do protocolo. Foram excluídos os que estavam de férias ou licença.

A coleta das informações se deu por meio de uma entrevista semiestruturada, utilizando um gravador, com horário previamente agendado com os profissionais em uma sala reservada mediante roteiro de entrevista que abordou os assuntos: conhecimento sobre o protocolo sepse, sua utilização e sobre os desafios e experiências exitosas de seu uso. As entrevistas gravadas foram transcritas na íntegra e analisadas com método de análise de conteúdo segundo Bardin $(2011)^{12}$. Nessa análise, o pesquisador busca compreender as características, estruturas ou modelos que estão por trás dos fragmentos de mensagens tomados em consideração, por meio de três fases fundamentais a seguir: pré-análise, exploração do material e tratamento dos resultados - a inferência e a interpretação, discutidas com a literatura pertinente. Foram finalizadas a partir do momento da saturação dos dados.

Participaram do estudo 14 enfermeiros, o anonimato foi respeitado utilizando-se um código alfanumérico (E1E, E1UTI...) para identificá-los em seus depoimentos.

O projeto foi aprovado pelo Comitê de Ética em Pesquisa com Seres Humanos (CEP) por meio da Plataforma Brasil da instituição de origem do estudo, com o CAAE no 2.894.469, conforme Resolução № 466/2012.

\section{RESULTADOS E DISCUSSÃO}

Dos enfermeiros participantes $79 \%$ eram do sexo feminino. A faixa etária predominante variou entre 30 a 39 anos, totalizando $57 \%$.

Em relação à especialização, todos os participantes afirmaram possuir um ou mais cursos de pós-graduação; contudo, 71\% 
haviam concluído e $29 \%$ estavam em processo de conclusão. Quanto ao tempo de graduação, 64\% dos entrevistados possuíam de 1 a 9 anos de graduação finalizada.

As unidades de registro obtidas com as entrevistas foram agrupadas e analisadas, originando quatro categorias a seguir: capacitação dos profissionais para manejo do protocolo; conhecimento acerca do protocolo sepse; desafios do enfermeiro no uso do protocolo; experiências exitosas: desfecho do paciente pós-protocolo.

\section{Capacitação dos profissionais para manejo do protocolo}

Pode-se observar nas falas a ausência da capacitação sobre o protocolo proveniente da instituição aos colaboradores, porém, conforme os relatos, nota-se uma colaboração entre os profissionais relativos ao ensino da utilização do documento entre os mesmos.

“[...]Na verdade, foi.... Não foi nem um treinamento peguei a rotina né peguei a rotina da unidade, três dias se eu não me engano e a enfermeira que tava aqui me passou a respeito dos protocolos clínicos. Foi isso."E2E

"Não, não recebemos treinamento nenhum só um checklist mesmo. O treinamento foi que, a cada protocolo sepse, a gente ia tentando aprimorar pra ver se conseguia cada protocolo fazer melhor; assim, ia dando certo e foi dando certo."E4E

"Treinamento específico não, só do dia a dia né do manejo do dia a dia do protocolo e no manuseio com paciente, mas assim especificamente não." E9UTI

Cabe ao enfermeiro, também, ter a autonomia para buscar em literaturas pertinentes conhecimento científico acerca do assunto, para que o cuidado e os procedimentos a serem realizados procedam de forma homogênea, promovendo segurança e qualidade da assistência ao paciente.

O processo de aprendizagem cooperativo fortalece o conhecimento e os relacionamentos interpessoais, uma vez que o trabalho da equipe de enfermagem se desenvolve de forma coletiva $^{13}$.

Há um considerado avanço em relação ao diagnóstico precoce; a identificação mais eficaz do microrganismo que possibilita o rápido início do tratamento e utilização de técnicas que possam estabilizar o paciente com quadro de sepse. Com isso, entendese que a detecção em tempo hábil é a etapa mais importante para o prognóstico positivo ${ }^{14}$.

Para a realização desta primeira fase, é necessário que os profissionais de saúde estejam capacitados para reconhecer a tempo os sinais e sintomas da doença, não deixando margem para sua evolução, bem como também, que a instituição assegure capacitação de seus colaboradores e disponha de protocolos que norteiem a assistência ${ }^{5}$.

\section{Conhecimento acerca do protocolo de sepse}

Os achados revelaram que os profissionais possuem conhecimento quanto à funcionalidade do protocolo, conforme fluxograma da instituição, porém deficiente em relação à doença. Mencionaram os procedimentos preconizados a serem realizados, o objetivo da existência do protocolo, conforme suas próprias experiências na utilização do documento. Houve dificuldades em caracterizar a sepse, bem como seus estágios.

"É um conjunto de informações né, de itens que a gente qualifica como se fosse o sepse, né." E1E

“É....tipo um pacote né que faz com que agilize o processo onde a gente identifica o foco, né, as... como é que se diz ... os sintomas lá da sepse e pode agilizar o processo, né." E2UTI

“Bom, na minha visão, protocolo sepse é um...como se fosse uma checklist para você seguir diante de um paciente que apresenta alguns sintomas que leva você a crer que ele está desenvolvendo uma sepse e a partir daquilo ali você vai excluindo algumas coisas e leva você a pensar que pode ser uma sepse você já se antecipa e é....já promove para o paciente um cuidado diferenciado, né, e direcionado. “E3E

"Protocolo sepse para mim é.... agilidade quanto a um quadro clínico de um paciente com risco iminente de choque séptico; então, protocolo sepse é eu diagnosticar eu evitar um choque séptico no paciente que chegue até mim." E5E

“É um protocolo que você em relação a uma infecção específica /né/ generalizada é independente de o foco ser o foco é um pouco direcionado, ou não.“E8UTI

A sepse difere-se de outras doenças por se apresentar com distintas situações clínicas dentro de um processo evolutivo do quadro, em uma mesma condição patológica e fisiológica. É uma resposta a uma inflamação sistêmica descontrolada, em que ocorrem mecanismos variados e complexos, envolvendo os mediadores inflamatórios, alteração na cascata de coagulação, anticoagulação e fibrinólise, resultando na hipoperfusão tissular, na morte celular com subsequente disfunção de múltiplos órgãos, levando ao óbito ${ }^{15}$.

Ao se deparar com um caso de sepse, o enfermeiro deve realizar as intervenções de modo imediato conforme for preconizado pelo protocolo institucionalizado. As medidas do pacote devem ser concluídas no tempo indicado, até as três primeiras horas, as quais consistem em: puncionar acesso venoso periférico calibroso; acionar o médico; contatar laboratório para a coleta 
de exames (hemocultura, gasometria, lactato, creatinina, bilirrubina, hemograma completo, cultura de sítios infecciosos); administrar antibiótico prescrito - o enfermeiro deve atentar para que o medicamento seja administrado após coleta de exames; administrar volume para hipotensão ${ }^{16}$.

Com isso, os protocolos assistenciais configuram-se como importante instrumento para reconhecimento precoce da sepse, pois asseguram maior segurança aos usuários e profissionais dos serviços de saúde; reduzem a heterogeneidade das ações de cuidado; empoderam profissionais para a tomada de decisão; são utilizados com indicadores para avaliação do processo e; facilitam a comunicação entre a equipe e coordenar o cuidado ${ }^{8}$.

Os dados encontrados corroboram a literatura, afirmando que os protocolos clínicos possuem a vantagem de serem ferramentas que direcionam melhor o cuidado, alinham os serviços que participam do fluxo e fazem que as intervenções sejam aplicadas rapidamente, além de auxiliar no diagnóstico precoce de sepse. Tal fato impacta diretamente na redução da mortalidade, com consequente refreamento do tempo de internação hospitalar, o qual eleva custos de tratamento ao hospital.

"Auxilia assim desde o momento da abordagem inicial da avaliação inicial do enfermeiro/ né/ porque ele já traz bem desenhado tudo aquilo que você vai fazer com o paciente... desde o momento da admissão de você identificar possíveis fatores de risco até a continuidade da assistência então é praticamente assim é... um manejo mesmo da assistência voltado para esse foco da sepse mesmo pro paciente." E3E

"O protocolo é uma forma...; é um tratamento bem adequado que a gente agiliza tudo entendeu, exames, é.... agilizar um antibiótico, agiliza tudo. Se for como uma sepse grave é....a....quando é uma sepse grave é o índice de melhora é....é notório, entendeu, depois de $48 \mathrm{~h}$ você já vê o paciente com a....a resposta bem nítida." E4E

"No controle e tratamento da sepse de imediato, na prevenção da piora do paciente e até na prevenção de um possível óbito que pode ser evitado." E8UTI

Os protocolos são padronizações estruturadas e bem delineadas que oferecem suporte na assistência clínica, pois dispõem de uma sequência temporal do cuidado, diagnóstico e tratamentos definidos. Objetiva oferecer qualidade no serviço, melhorando os cuidados de saúde. Para realização desse processo, é necessária uma mobilização e envolvimento de gestores, coordenadores e colaboradores, para compreenderem a importância de implementar essas padronizações e, consequentemente, aderir as estratégias propostas, entendendo que o objetivo é tornar o cuidado mais seguro ${ }^{9}$.
Segundo estudos, devido as relevantes vantagens que os protocolos possuem, observaram-se que, nos EUA, esses foram bem disseminados e adotados no país, diferentemente do Brasil, que apresenta uma escassa implementação de protocolos. A pesquisa teve como objetivo avaliar o grau de cumprimento de medidas de um protocolo clínico para manejo da sepse e analisar o impacto das intervenções nas taxas de sobrevivência. Conclui que, apesar de o cumprimento das intervenções ter sido baixo, foi observado que houve uma queda da mortalidade dos pacientes acometidos por sepse e menor duração de internação hospitalar 5 .

\section{Desafios do enfermeiro no uso do protocolo}

Observam-se, nas falas, muitos desafios que dificultam o desdobramento do protocolo quando acionado; entre elas, foi citada a restrição da assistência de enfermagem em iniciar o pacote de três horas, pois não é da atribuição do enfermeiro a prescrição das atividades do protocolo; dificuldades em cumprir tempo de administração do antibiótico por demora de prescrição ou envio da farmácia; demora da resposta dos serviços acionados (laboratório, farmácia, cirurgião) e demora do diagnóstico médico de sepse.

"Acho que o grande desafio também é em relação à parte médica na identificação e no manejo correto, por que nem sempre eles identificam, vem...tipo, por medo eles veem sepse aonde não, às vezes nem tem e aí acaba que prejudica nosso trabalho, mas...eu acho que é um dos grandes desafios né, o treinamento médico também[...]". E2E

"Os desafios eu acredito que, como infelizmente a gente depende muito da questão de laboratório /né/, de cirurgião pra vim puncionar o acesso central e nem sempre a gente consegue agilizar e estar dentro daquela 1 hora. [...] Mas por depender de laboratório, cirurgião e esses outros fatores infelizmente acabam sendo um desafio [...]" E2UTI

“[...] As dificuldades, elas ainda existem é porque às vezes você aciona o protocolo mas o médico não concorda com sua conduta, então assim então você fica impossibilitado de agir por que você abre o protocolo mas você não tem como prescrever um antibiótico, você não tem como agir então, assim , você tem que contra argumentar e mostrar fatos reais daquilo, da sua conduta, daquilo que você tá propondo pro paciente mas de uma maneira geral é... [...]" E3E

"Os desafios ainda é muitos é com os médicos, porque tem muito médico que quer abrir um protocolo só pra poder iniciar o antibiótico, sem critérios nenhum pra protocolo. [...] Então o nosso desafio ainda é colocar na cabeça dos médicos que protocolo sepse não significa só iniciar antibiótico. [...]"E4E 
"É....os desafios é que a enfermeira que tem que acionar todos os responsáveis por que fazem parte do protocolo é....devia ser uma forma mais uma ter uma forma mais ágil para isso até porque a gente precisa dar assistência ao paciente precisa fazer os procedimentos com pacientes [...] E4UTI

Caso haja entraves para a concretização do que é proposto pelo protocolo, ou ainda não houver adesão por parte da equipe, as ações de melhoria são discutidas e elaboradas para sanar essas e outras dificuldades ${ }^{9}$.

Os próprios profissionais em seus relatos sugerem algumas alternativas de soluções para alguns desafios que foram elencados, conforme experiências vivenciadas com o uso do documento; apontam a necessidade de educação permanente voltada para a classe médica acerca do protocolo sepse, bem como, uma melhoria na comunicação para o acionamento dos serviços, para que, quando houver uma abertura de protocolo, haja uma sinalização como um start para todos os envolvidos ao mesmo tempo.

As falas denotam a Prática Baseada em Evidência, a qual é definida como uma abordagem de soluções de problemas, de forma consciente, explícita e criteriosa, da melhor evidência disponível na tomada de decisão para prestar o melhor cuidado em saúde. Requer uma associação da literatura à experiência clínica do profissionali ${ }^{17}$.

Experiências exitosas: desfecho do paciente pós-protocolo.

Com a implementação do protocolo nas situações de sepse, todos os enfermeiros relataram uma real eficácia no bom prognóstico dos pacientes acometidos pelo agravo. Houve regressão do início do quadro, reabilitação hemodinâmica, consequentes do rápido reconhecimento e ações imediatas pertinentes.

“[...]É....experiências exitosas é na questão de poder lidar mais rápido, de evitar que essa sepse acabe evoluindo para um choque." E2UTI

“Bom a experiência boa é você poder ver que é.... o paciente é mesmo nas primeiras horas de cuidado ele já apresenta uma melhora ele já apresenta sinais de chance de ser revertido o quadro então isso pra gente da assistência isso é muito gratificante[...]E3E.

"[...] mas de experiência exitosa que você pode ter um resultado satisfatório se você seguir o protocolo os tempos certos, né? Assim você pode ter uma eficácia melhor e conseguir que o paciente saia da sepse, do grave". E5UTI

"[...] Às vezes tenho pegado pacientes muito graves, que chegaram muito graves com pressão chocados mesmo, conseguiram responder bem ao tratamento do protocolo sepse quando foi atendido a tempo e a hora de imediato, quando não demorou iniciar antibiótico, não demorou coleta de exames, não demorou saber resultados de exames, não demorou a achar o foco da infecção, foi muito bom, por que auxilia muito, evita muito o óbito do paciente." E8UTI

Koenig et al. (2017) realizaram um estudo de análise descritiva retrospectiva que avaliou o impacto da utilização de um pacote de medidas para sepse nas unidades de um hospital terciário. Foram estudados 167 pacientes, os quais receberam as intervenções do pacote de três horas, que consistia em coletar lactato e culturas, iniciar antibiótico ao diagnóstico de sepse nas primeiras horas. Conclui que, com o uso do protocolo para manejo da sepse, houve uma menor mortalidade dos pacientes, menor necessidade de internação em UTI e menor permanência nas unidades do hospital ${ }^{18}$.

Outro estudo realizado em um hospital em Porto Rico teve como objetivo verificar a incidência de sepse e suas consequências. Foi realizado um estudo retrospectivo observacional. A análise mostrou altas taxas de incidência e mortalidade pela doença. A pesquisa concluiu que, diante dos dados coletados, a instituição adotasse protocolos para reduzir a mortalidade, com estratégias adequadas para diagnóstico precoce da sepse ${ }^{19}$.

Diament et al. (2011) afirmam, em seu estudo, que, para haver chances de sobrevivência dos pacientes acometidos por sepse, faz-se necessária a existência de um protocolo clínico, pois ele direciona o cuidado e faz que as intervenções sejam aplicadas rapidamente, bem como o reconhecimento do agravo seja logo identificado. O que reforça a suma importância de se implementarem protocolos nas unidades hospitalares ${ }^{20}$.

A preocupação com os custos dispensados ao cuidado em saúde nas redes hospitalares privadas vem-se intensificando ao longo do tempo. A ideia principal é o gerenciamento de qualidade a fim de que, cada vez mais se possam diminuir esses custos provenientes de internações hospitalares prolongadas por infecções e agravos e, ao mesmo tempo, ofertar melhorias da assistência ao cliente. Com isso, trabalha-se com padronizações do cuidado, que agrega participação de todos os interessados, trazendo consigo o conceito de melhoria contínua ${ }^{21}$.

\section{CONSIDERAÇÕES FINAIS}

Por meio deste estudo, foi possível evidenciar a atuação do enfermeiro diante de pacientes com quadro séptico. Infere-se que as ações de enfermagem não se restringem ao cumprimento do protocolo e da realização das medidas de intervenção; faz-se necessária uma avaliação minuciosa desse paciente em relação à resposta de seu organismo ao que foi administrado.

Nessa assistência, incidem ainda os desafios que eles enfrentam para que o processo tenha início e fim e não seja interrompido por qualquer eventualidade, como demora dos serviços 
acionados. Reforça que o protocolo não é apenas mais um documento da assistência, e sim uma ferramenta importante para prestar o melhor cuidado de enfermagem.

Para o hospital em que foi realizado o presente estudo, a adesão da utilização do protocolo remete a um atendimento de qualidade e segurança ao paciente, em que se configura estar adequado em um dos requisitos do processo de acreditação hospitalar que almeje alcançar; menor de tempo de internação hospitalar - o que reduz custos desse processo -, e consequente melhoria da imagem e competitividade entre os outros hospitais particulares.

\section{REFERÊNCIAS}

1. Viana RAP, Machado FR, Souza JLA. Sepse, um problema de saúde pública: a atuação e colaboração da enfermagem na rápida identificação e tratamento da doença. 2.ed. São Paulo: COREN-SP; 2017.

2. Moreno RP, Martin GS. Sepsis without borders. Intensive Care Med. 2014 Nov [acesso 2018 Nov 11]; 40(2): 272-274. Disponível em: https://link.springer. com/content/pdf/10.1007\%2Fs00134-013-3176-5.pdf. doi: 10.1007/s00134013-3176-5.

3. Taniguchi LU, Pires EMC, Vieira JM Jr, Azevedo LCP. Critérios para síndrome de resposta inflamatória sistêmica e predição de mortalidade hospitalar em pacientes críticos: estudo retrospectivo de coorte. Rev. bras. ter. intensiva [internet]. 2017 Set [acesso 2018 Nov 10]; 29(3): 317-324. Disponível em: http://www.scielo.br/scielo.php?script=sci_arttext\&pid=S0103507X2017000300317\&lng=en. doi: http://dx.doi.org/10.5935/0103$507 x .20170047$.

4. Instituto Latino-Americano de Sepse. Sepse: um problema de saúde pública. Brasília: CFM; 2015.

5. Laguna-Pérez A, Chilet-Rosell E, Delgado LM, Alvarez-Dardet C, Uris SJ, MuñozMendoza CL. Observância e efetividade das intervenções de um protocolo clínico utilizado para pacientes com sepse grave e choque séptico de uma Unidade de Cuidados Intensivos da Espanha. Rev. Latino-Am. Enfermagem [internet]. 2012 Ago [acesso 2018 Nov 11]; 20(4): 635-643. Disponível em: http://www.scielo. br/scielo.php?script=sci_arttext\&pid=S0104-11692012000400002\&lng=en . DOI: http://dx.doi.org/10.1590/S0104-11692012000400002.

6. Contrin LM, Paschoal VDA, Beccaria LM, Cesarino CB, Lobo SMA. Qualidade de vida de sobreviventes de sepse grave após alta hospitalar. Rev. Latino-Am. Enfermagem. 2013; 21(3): 795-802.

7. Carvalho PRA, Trotta EA. Avanços no diagnóstico e tratamento da sepse. J. Pediatr. (Rio J.) [Internet]. 2003 Nov [acesso 2018 Nov 15] ; 79( Suppl 2): S195-S204. Disponível em: http://www.scielo.br/scielo.php?script=sci_ arttext\&pid=S0021-75572003000800009\&Ing=en. doi: http://dx.doi. org/10.1590/S0021-75572003000800009.

8. Pimenta CAM, Jensen R, Shimoda GT, Nishi FA, Amorim AF, Lopes CT. Guia para construção de protocolos assistenciais de enfermagem. São Paulo: CORENSP. 2015.

9. Medeiros AP, Amaral CFL, Laurindo MC, Souza DA, Nadai TR. Implementação de um protocolo clínico gerenciado de sepse grave e choque séptico. Rev Qualidade HC. 2015; 10(5): 1-12.

10. Ferreira RGS, Nascimento JL. Intervenções de enfermagem na sepse: saber e cuidar na sistematização assistencial. Ver Saúde e Desenvolvimento. 2014 JulDez; 6(3): 51-59.

11.Cintra EA, Nishide VM, Nunes WA. Assistência de enfermagem ao paciente gravemente enfermo. 2.ed. São Paulo: Atheneu; 2013.
13. Cogo ALP. Cooperação versus colaboração: conceitos para o ensino de enfermagem em ambiente virtual. Rev. bras. enferm. [Internet]. 2006 Out [acesso 2018 Dez 1] ; 59( 5 ): 680-683. Disponível em: http://www.scielo.br/ scielo.php?script=sci_arttext\&pid=S0034-71672006000500016\&Ing=en. doi: doi.org/10.1590/S0034-71672006000500016.

14. Barreto MFC, Dellaroza MSG, Kerbauy G, Grion CMC. Sepsis in a university hospital: a prospective study for the cost analysis of patients' hospitalization. Rev. esc. enferm. USP [Internet]. 2016 Mar-Abr [acesso 2018 Nov 12] ; 50(2): 302-308. Disponível em: http://www.scielo.br/scielo.php?script=sci_ arttext\&pid=S0080-62342016000200302\&Ing=en. DOI: http://dx.doi. org/10.1590/S0080-623420160000200017.

15. Davida CM, Faria Neto HC. Sepse - Da Bancada à Beira do Leito. São Paulo: Thieme Revinter; 2007.

16. Dellinger RP, Levy MM, Rhodes A, Annane D, Gerlach H, Opal SM et al. Campanha de sobrevivência à sepse: Diretrizes internacionais para tratamento de sepse grave e choque séptico: 2012. CCM Journal. 2013; 14(2): 567-571.

17. Camargo FC, Iwamoto HH, Galvão CM, Pereira GA, Andrade RB, Masso GC. Competences and Barriers for the Evidence-Based Practice in Nursing: an integrative review. Rev. Bras. Enferm. [Internet]. 2018 Ago [acesso 2018 Nov 15] ; 71(4): 2030-2038. Disponível em: http://www.scielo.br/scielo. php?script=sci_arttext\&pid=S0034-71672018000402030\&lng=en. doi: http:// dx.doi.org/10.1590/0034-7167-2016-0617.

18. Koenig A, Picon PD, Feijó J, Silva E, Westphal GA. Estimativa do impacto econômico da implantação de um protocolo hospitalar para detecção e tratamento precoce de sepse grave em hospitais púbicos e privados do sul do Brasil. Rev. bras. ter. intensiva [Internet]. 2010 Set [acesso 2018 Nov 4]; 22(3): 213-219. Disponível em: http://www.scielo.br/scielo.php?script=sci_ arttext\&pid=S0103-507X2010000300001\&Ing=en. doi: http://dx.doi. org/10.1590/S0103-507X2010000300001.

19. Morales ST, Ramos S, Lara GY, Torres CH, Vera QA, Miranda SR et al. Manatí Medical Center Sepsis Management Epidemiological Study. Bol Asoc Med. 2015 Apr; 107(2): 34-9.

20. Salomão R, Diament D, Rigatto O, Gomes B, Silva E, Carvalho NB et al . Diretrizes para tratamento da sepse grave/choque séptico: abordagem do agente infeccioso - controle do foco infeccioso e tratamento antimicrobiano. Rev. bras. ter. intensiva [Internet]. 2011 Jun [acesso 2018 Nov 1]; 23(2): 145-157. Disponível em: http://www.scielo.br/scielo.php?script=sci_arttext\&pid=S0103507X2011000200006\&lng=en. doi http://dx.doi.org/10.1590/S0103$507 \times 2011000200006$.

21. Lagioia UCT, Ribeiro Filho JF, Falk JA, Libonati JJ, Lopes JEG. (2008). A gestão por processos gera melhoria de qualidade e redução de custos: o caso da unidade de ortopedia e traumatologia do hospital das clínicas da Universidade Federal de Pernambuco. Rev. contab. Finanç; 19(48): 77-90. doi: https://dx.doi. org/10.1590/S1519-70772008000300007.

12. Bardin L. Análise de conteúdo. São Paulo: Edições 70; 2011.

Como citar este artigo/How to cite this article:

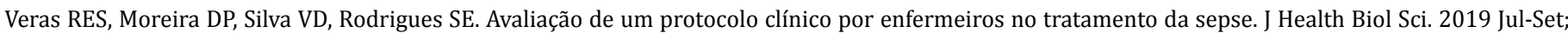
7(3):292-297. 